\title{
Performance Analysis Of Electrical Properties Of Resin Transfer Molded Banana Leaf Reinforced Polymer Composites
}

T. Rajamanikandan ${ }^{1 *}$, Assistant Professor, Department of EEE, Kongunadu College of Engineering and

Technology,

Trichy, Tamilnadu, India

E-mail:mvtbharatham@gmail.com

Dr.S. Banumathi ${ }^{2}$,

Professor, Department of EEE, M.Kumarasamy College of Engineering,

Karur, Tamilnadu, India

Dr.R. Asokan ${ }^{3}$

Professor, Department of ECE, Kongunadu College of Engineering and Technology

Trichy, Tamilnadu, India

*1 mvtbharatham@gmail.com

\begin{abstract}
An investigation of the electrical characteristics of banana leaf reinforced Polymer composites made by resin transfer molding (RTM) has been carried out, with special emphasis on the effects of fibre loading, frequency and temperature. Every parameter, including the dielectric constant $\left(\varepsilon_{0}\right)$, dissipation factor $(\tan \delta)$, loss factor $\left(\varepsilon_{00}\right)$, and conductivity, increases with increasing fibre concentration over the whole frequency range. A minimum fibre content of $50 \%$ is required for composites to achieve excellent performance values. This increase is large at low frequencies, minimal at middle frequencies, and negligible at extremely high frequencies, according to the results of the study. At low frequencies, the volume resistivity fluctuates in response to fibre loading, while at high frequencies, the resistivity blends together. When the temperature rises, the dielectric constant values rise as well, however once the glass transition temperature is reached, the dielectric constant values fall. This fluctuates depending on the amount of fibre present. Finally, an attempt is made to establish a relationship between the experimental value of the dielectric constant and theoretical expectations.
\end{abstract}

Keywords: Electrical properties; Resin transfer molding; Banana leaf; Polymer composites; dielectric.

\section{INTRODUCTION}

Recently, both academic and industrial researchers have shown a strong interest in natural fibres in both macro and microforms as reinforcement for polymers. This has occurred in both the industrial and academic sectors during the previous few decades. Many different 
fibers and plants are used as reinforcements in the production of composites. Some of the most commonly used fibers and plants are flax, hemp, oil palm, coir, jute, kenaf, and banana [1-6]. Natural fibre composites have several advantages over synthetic fibre reinforced composites, including the use of renewable raw materials, low cost, light weight, and high specific strength and stiffness. Natural fibre composites are also more environmentally friendly than synthetic fibre reinforced composites. These composites can be made using a variety of fabrication techniques, including resin transfer molding (RTM), compression molding (CM), and manual lay-up. Throughout this essay, we'll talk about a breakthrough processing technology that can be used to fabricate high-performance composites. Our laboratory has previously showed that RTM can be used to fabricate randomly oriented short banana leaf-Polymer composites with excellent fiber-matrix adhesion using short banana leaves. However, the vast bulk of research in the field of RTM has concentrated on the visualization of the flow front, modeling of void creation, and numerical simulations of curing using natural and synthetic fibres, rather than on the process itself [6-12].

In recent years, there has been an increase in interest in the creation of electrically conductive polymeric composite materials. In order to successfully employ these materials, it is essential to understand more information about their dielectric constant, volume resistivity, and loss factor. The composition, chemical structure, physical texture, and shape of the materials all influence their physical and mechanical properties. A large number of studies on the electrical properties of filler reinforced polymers have been reported in the literature. Natural rubber and ethylene vinyl acetate that have been packed with conductive carbon black and short carbon fibre have excellent shielding properties [13-16]. To better understand the electrical characteristics of fibre reinforced rubber composites, Thomas and colleagues undertook considerable study. In their research, Cabral et al. looked at the dielectric characteristics of short jute fiber-reinforced polypropylene composites. It was discovered that when the fibre content reached a threshold level, the dependence of dielectric properties on fibre loading shifted. Many studies on the electrical properties of thermoset composites with filler reinforcement have been published in the past few years. Find out how well epoxy composites with 0.004 - 0.4 weight percent single-walled or multi-walled carbon nano tubes carry electricity and heat. An investigation into the electrical properties of glass fibre reinforced epoxy composites containing $0.4 \%$ amino-functionalized double-wall carbon nano tubes was conducted [17-19]. 
An anisotropic conductivity was observed in the composite, with conductivity in the plane being one order of magnitude larger than conductivity out of plane for the material. The electrical properties of randomly distributed banana leaf-Polymer composites made via RTM have not, however, been thoroughly investigated. In this respect, the purpose of this paper is to shed light on the effects of fibre loading, frequency, and temperature on the electrical properties of banana leaf-Polymer composites.

\section{EXPERIMENTAL}

\subsection{MATERIALS}

ICAR- National Research Centre for Banana, Tamilnadu, India provided the banana Banana leafs. BohriAli Enterprises, India, supplied cobaltnapthanate, and methyl ethyl ketone peroxide with a purity of $96 \%$.

\subsection{PREPARATION OF COMPOSITES}

Isophthalic polymer composites with banana leaf fibre reinforcement have been created using RTM technology. The banana leaf fibers were randomly oriented and had an ideal fibre length of $30 \mathrm{~mm}$, which was used to fill the mould. Injection of the Polymer resin was carried out at an finest pressure of $1 \mathrm{~kg}$ per $\mathrm{cm}^{2}$ in conjunction with $1 \%$ cobaltnapthanate and $1 \%$ methyl ethyl ketone peroxide, with the mould being closed and clamped after each injection. Vacuum was provided simultaneously with the resin impregnation in order to minimize the creation of voids. At room temperature, the mould should be sealed to keep out air throughout the curing process $\left(28^{\circ} \mathrm{C}\right)$. This was followed by de-molding and post-curing the composites for 3 hours in a free standing mode at an temperature of $80^{\circ} \mathrm{C}$.

\subsection{ELECTRICAL ANALYSIS OF BANANA LEAF RTM COMPOSITE}

DEA 2970 dielectric analyzer equipped with a liquid nitrogen cooling accessory was used for the dielectric relaxation experiments. Parallel plate sensors were employed to examine the samples, which were exposed to a $300 \mathrm{~N}$ force in order to ensure appropriate contact between the specimen and the electrodes during the testing process. The studies were carried out at frequencies ranging from $1 \mathrm{~Hz}$ to $400 \mathrm{kHz}$ and at temperatures ranging from $28^{\circ} \mathrm{C}$ to $150^{\circ} \mathrm{C}$. A sinusoidal voltage was used to generate an alternating electric field, which was then reversed. In this study, the discs under investigation have a diameter of $25 \mathrm{~mm}$ and a one millimeter thickness. 
It is possible to derive the volume resistivity (q) from the resistance by use the equation.

$$
\rho=\text { RA-t }
$$

where $\mathrm{V}$ represents volume resistivity, $\mathrm{R}$ represents resistance, A represents the crosssectional area of the sample, and trepresents the thickness of the sample

\section{RESULTS AND DISCUSSION}

It is defined as the ratio of the capacitance of a material-containing condenser to that of the same material containing condenser in vacuum when a material is used as the dielectric constant of a material. Figure 1 depicts the dielectric constant of banana leaf-polymer composites as a function of frequency for various frequencies. Fiber content increases the dielectric constant in the sequence Polymer R20, R30, R40, R50, and this is true for all frequencies over the whole frequency range. Found a similar tendency in mineral-filled epoxy composites, which was confirmed [20].

The interfacial, orientation, atomic, and electronic polarizations present in the material play a significant role in determining the $\varepsilon_{0}$ values of composites, as well as their composition. It is possible for interfacial polarization to arise as a result of the matrix and filler having differing conductivities or polarizations from one another. The orientation of the filler and the interfacial polarizations are also affected by the amount of filler present. The considerable increase in $\varepsilon_{0}$ with increasing fibre loading was due to an increase in orientation and interfacial polarization produced by the presence of cellulose polar groups in natural fibers (which they found to be true) [21].

In Figure 2, the relationship between the composite dielectric constant and the matrix dielectric constant is shown as a function of fibre content, and this is illustrated more clearly. While this is true in Polymer, the $\varepsilon_{0}$ value is modest since it only takes into account immediate atomic and electronic polarizations, which are less important.

Also revealed is that, for a given fibre loading, $\varepsilon_{0}$ exhibits high values at lower frequencies, which is related to the decrease in orientation polarization with increasing frequency. Complete orientation of molecules can only be achieved at lower frequencies, and orientation polarization takes longer to reach equilibrium static field values than electronic or atomic polarizations, indicating that it is more difficult to achieve complete orientation. The result is 
that when frequency increases, $\varepsilon_{0}$ drops as a result of the time delay in polarization orientation [22].

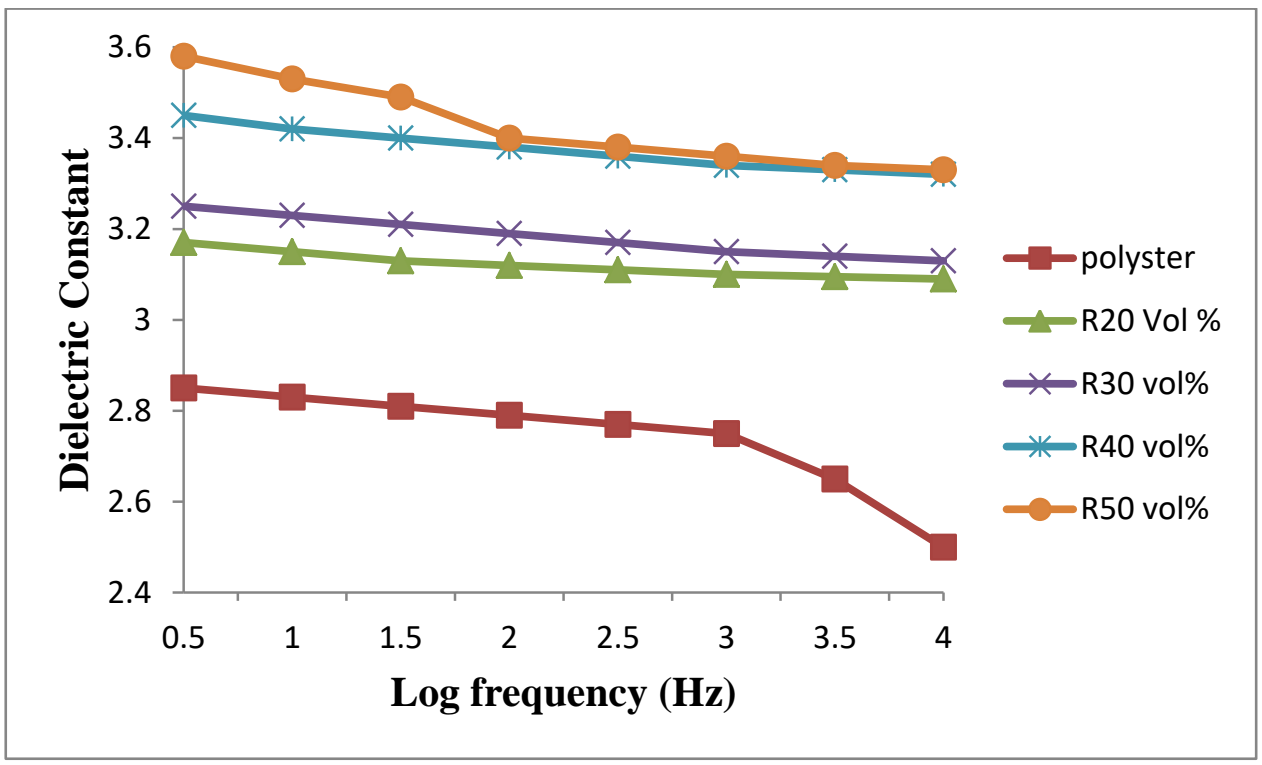

\section{Figure 1.Banana leaf-Polymer composites in different dielectric constant with different frequencies at $30^{\circ} \mathrm{C}$.}

It is defined as the ratio of the capacitance of a material-containing condenser to that of the same material-containing condenser in vacuum when a material is used as the dielectric constant of a material. Figure 1 depicts the dielectric constant of banana leaf-polymer composites as a function of frequency for various frequencies. Fiber content increases the dielectric constant in the sequence Polymer R20 R30 R40 R50, and this is true for all frequencies over the whole frequency range.

The interfacial, orientation, atomic, and electronic polarizations present in the material play a significant role in determining the $\varepsilon_{0}$ values of composites, as well as their composition. It is possible for interfacial polarization to arise as a result of the matrix and filler having differing conductivities or polarizations from one another. The orientation of the filler and the interfacial polarizations are also affected by the amount of filler present. The considerable increase in $\varepsilon_{0}$ with increasing fibre loading was due to an increase in orientation and interfacial polarization produced by the presence of cellulose polar groups in natural fibers.

In Figure 2, the relationship between the composite dielectric constant and the matrix dielectric constant is shown as a function of fibre content, and this is illustrated more clearly. While this is true in Polymer, the $\varepsilon_{0}$ value is modest since it only takes into account immediate atomic and electronic polarizations, which are less important. 
Also revealed is that, for a given fibre loading, $\varepsilon_{0}$ exhibits high values at lower frequencies, which is related to the decrease in orientation polarization with increasing frequency. Complete orientation of molecules can only be achieved at lower frequencies, and orientation polarization takes longer to reach equilibrium static field values than electronic or atomic polarizations, indicating that it is more difficult to achieve complete orientation. The result is that when frequency increases, $\varepsilon_{0}$ drops as a result of the time delay in polarization orientation [1].

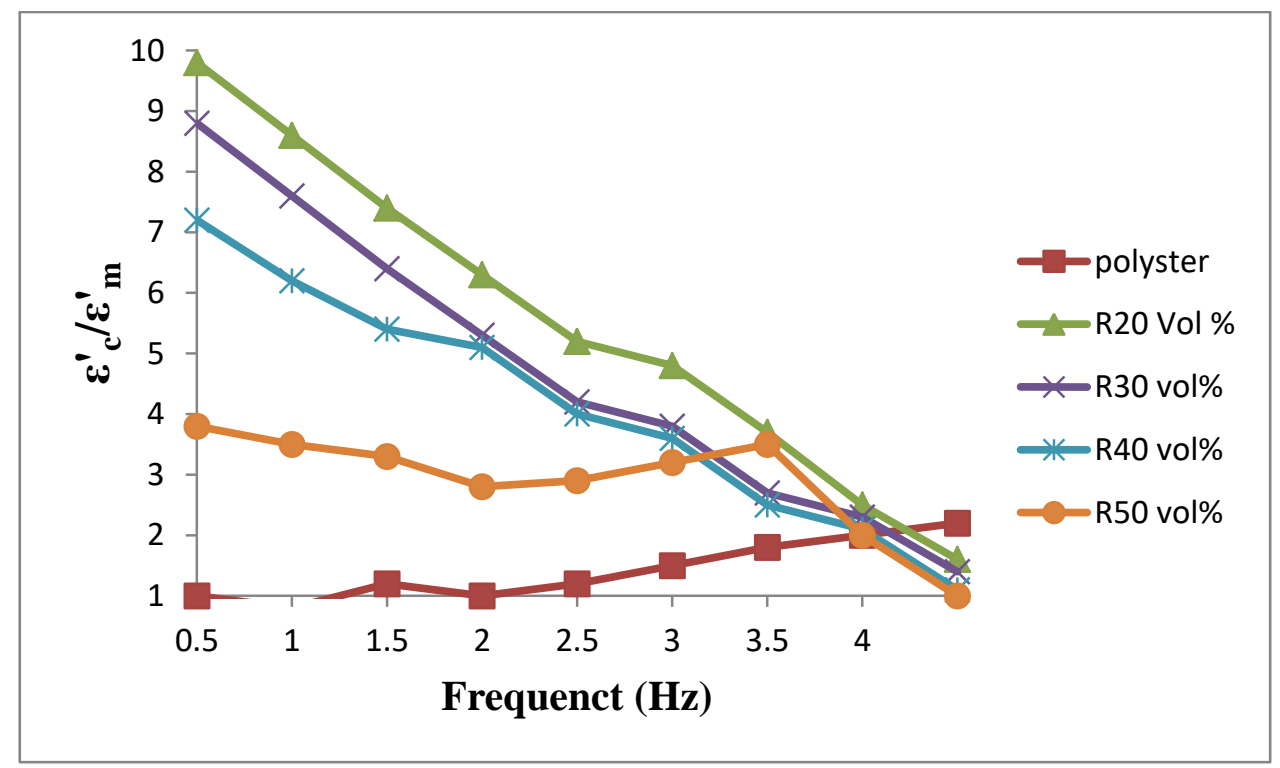

Figure 2.Dielectric constant of banana leaf composites with different $\mathrm{RTM}$ at $30^{\circ} \mathrm{C}$.

The orientation of the filler and the interfacial polarizations are also affected by the amount of filler present. When fibre loading was increased, there was a significant increase in orientation and interfacial polarizations, which was attributed to increased orientation and interfacial polarizations brought about by the presence of cellulose polar groups in natural fibers [22]. In Figure 2, the relationship between the composite dielectric constant and the matrix dielectric constant is plotted as a function of fibre content, and this is illustrated more clearly. While this is true in Polymer, the $\varepsilon_{0}$ value is small because it only takes into account immediate atomic and electronic polarizations, which are less important. 


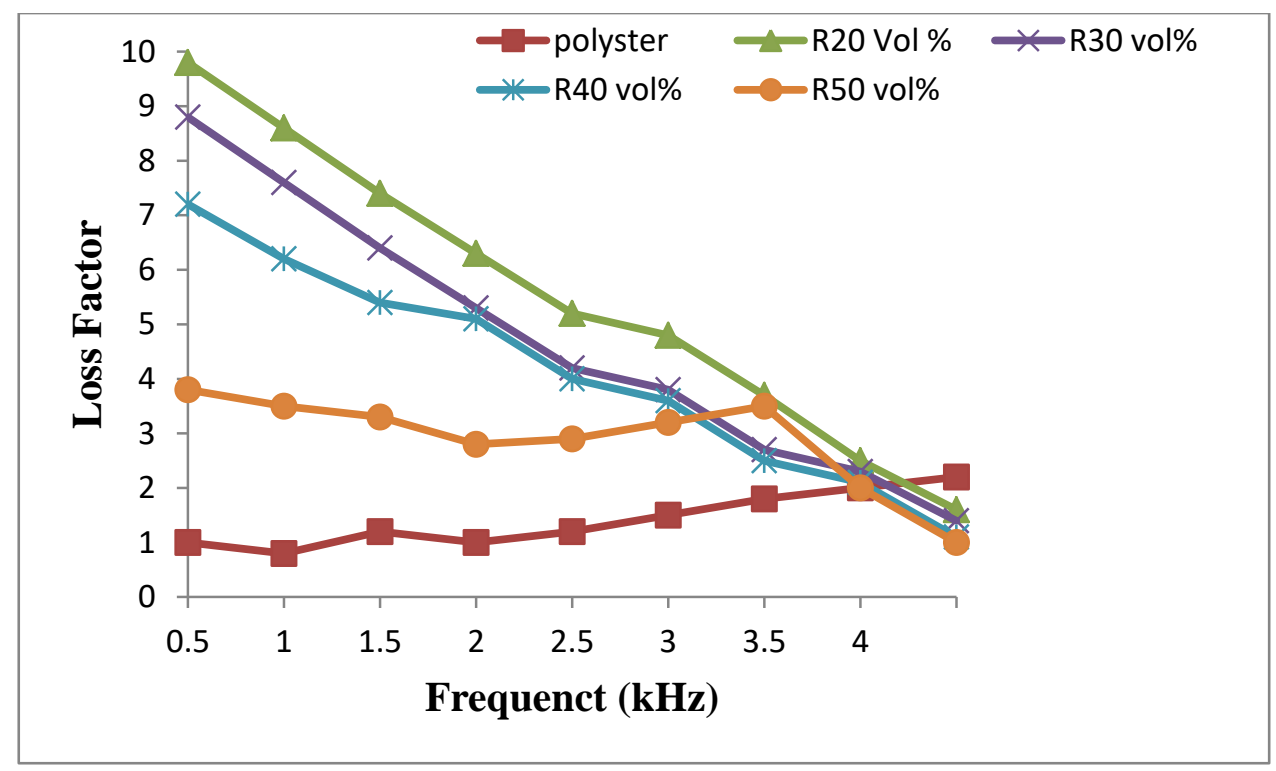

Figure 3.Loss factor of banana leaf-Polymer composites with different frequency at $30^{\circ}$ C.

Furthermore observed is the that, for a given fibre loading, $\varepsilon_{0}$ exhibits high values at lower frequencies, which is due to the decrease in orientation polarization with increasing frequency. Complete orientation of molecules can only be achieved at lower frequencies, and orientation polarization takes longer to reach equilibrium static field values than electronic or atomic polarizations, indicating that it is more difficult to achieve complete orientation. So as frequency is increased, $\varepsilon_{0}$ decreases due to the time delay in the orientation of the polarization vector.

The loss factor $\left(\varepsilon_{00}\right)$ is a mathematical representation of the average power factor over a specified time period that is used in the energy industry to express transmission and distribution losses. It is derived from the average power factor over a specified time period. As shown in Figure 3, composites exhibit fluctuation in the number $\varepsilon_{00}$ as a function of frequency. Because of this, the $\varepsilon_{00}$ is highest in the lower frequency zone and highest for composites with a fibre concentration of $50 \%$ at a particular frequency, as can be seen.

The value of $\varepsilon_{00}$ decreases rapidly as the frequency of occurrence increases, but the slope of the curve increases as the frequency increases. This demonstrates that the $\varepsilon_{00}$ is dependent on the fibre content in the lower frequency range, whereas the $\varepsilon_{00}$ is absent at higher frequencies due to the polarization of the fibers in the higher frequency range. The amount of fibre in the composite increases the heterogeneity of the composite, resulting in an increase in the amount of polarization in the composite. This phenomenon, which is similar to interfacial 
polarization, is caused by ionic conduction and dipole orientation in the same way. Additional to this, a multiphase system composed of banana leaf and polymer contains two dielectrically distinct materials: the polar and hydrophilic banana leaf and the non-polar and hydrophobic polymer, respectively. A system with discrete inter-phase will be loss at low frequencies and exhibit significant interfacial polarization if the inter-phase is discrete.

The dissipation factor $(\tan \delta)$ is defined as the relationship between the amount of electrical power wasted by a material and the total amount of electrical power flowing through the circuit.

The dissipation factor can be calculated with the help of the equation.

$$
\tan \delta=\varepsilon^{\prime} / \varepsilon^{\prime}
$$

Where the loss factor is $\varepsilon_{00}$ and the dielectric constant is $\varepsilon_{0}$.

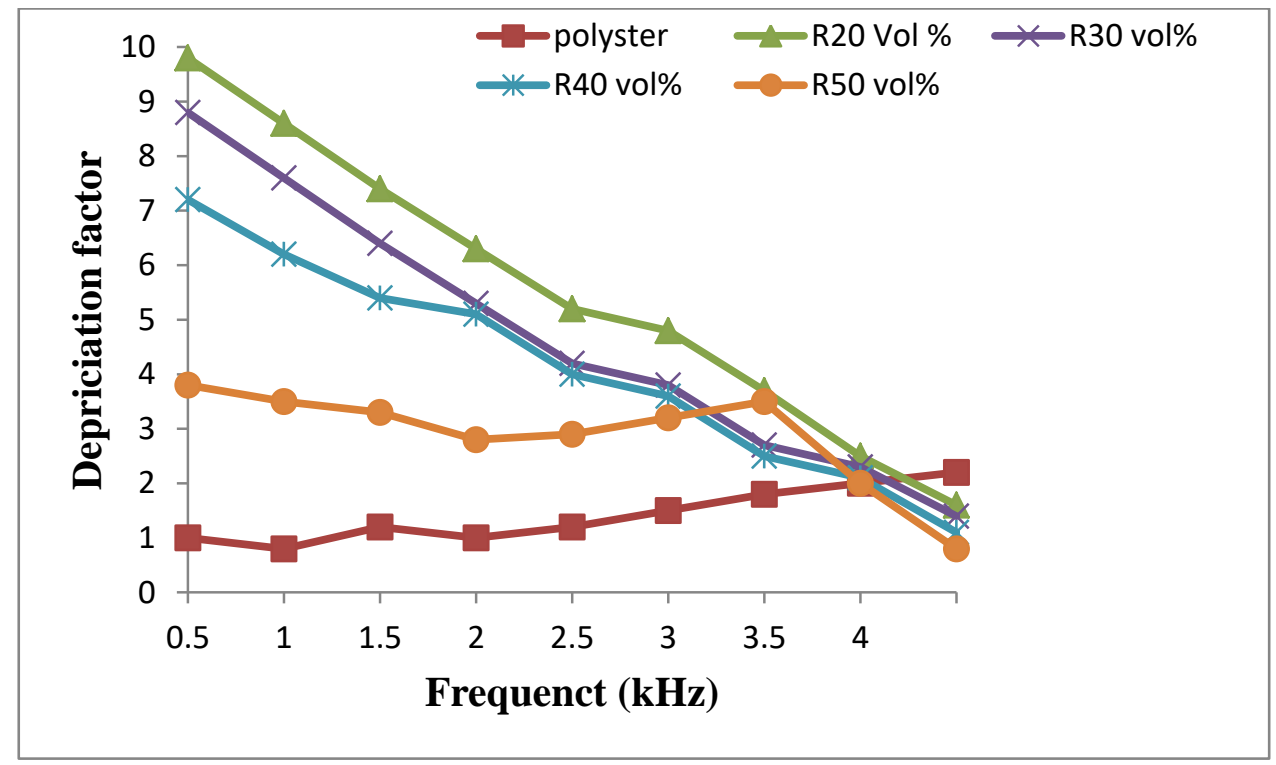

\section{Figure 4.Dissipation factor of banana leaf-Polymer composites with different frequencies at $30^{\circ} \mathrm{C}$.}

In order to determine the amount of electrical energy converted to heat in an insulator, it is necessary to measure the loss tangent of the material under consideration. Due to the heat generated, the temperature of the insulator rises, hastening its decomposition. For various fibre loadings in composites, Figure. 4 shows the influence of the dissipation factor as a function of frequency for various fibre loadings. It has been discovered that the dissipation factor decreases as the frequency of the event grows in frequency. With an increase in the fibre content, there is a significant shift in the low frequency zone, with Polymer being the 
lowest frequency and R50 being the highest frequency for a given frequency. However, as can be seen from the loss factor, the tan curves become closer together as the frequency range increases. As the amount of fibre in a cell grows, the number of polar groups grows as well, resulting in an increase in orientation polarization. This has the effect of increasing the dissipation factor. Additionally, because of their ability to absorb moisture, the addition of fibers improves current flow through the amorphous region of the material. As frequency increases, it becomes more difficult to polarize polar groups, and as a result, the dissipation factor decreases significantly.

A comparison of volume resistivity variations for composites with different fibre loadings can be seen in Figure. 5. It's important to note that the resistivity of the volume decreases as the frequency of the signal increases. This decrease is caused by the interfacial polarization that occurs as a result of the system's heterogeneity, which is responsible for the decrease. Also demonstrated is that the volume resistivity varies with fibre loading at a specific frequency, with the variation being the most pronounced in the low frequency range. This suggests that when banana leaves are added to a matrix, the conductivity changes as a result of the presence of polar groups, which allow current to flow through the matrix to the outside.

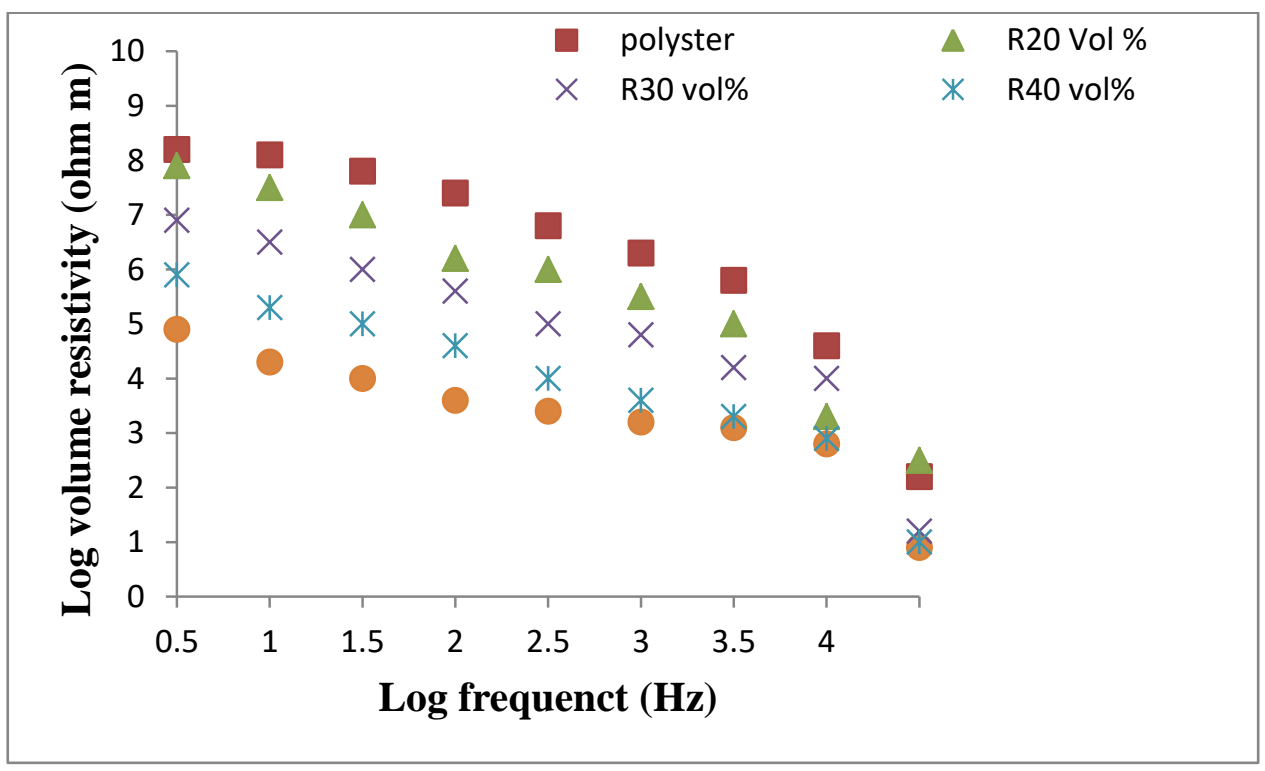

\section{Figure 5.Volume resistivity of the banana leaf composites with different fiber loading and different frequency at $30^{\circ} \mathrm{C}$.}

Aside from that, elongated fibers and flakes have the potential to significantly increase electrical conductivity. When moisture is present, it is well established that the majority of current flows through the crystalline areas of polymers and that current flow through the 
amorphous regions occurs when moisture is absent [23]. The hydroxyl groups in the banana leaf absorb moisture, increasing the conductivity of the matrix as a result. Figure 6 depicts the electrical conductivity variation of composites with fibre loading as a function of frequency for composites with fibre loading. The conductivity of the composite material increases in proportion to the increase in frequency and fibre loading.

It is greatest for composites that have a fibre composition of $50 \%$ or more by volume of the composite. Figure 7 illustrates how this is due to an increase in the number of polar groups present in banana leafs as a result of an increase in polar groups present in banana leafs. Conductivity in composites is also dependent on the dispersion of fibers within the material. When the volume fraction of fibers is small, there is a chaotic dispersion of fibers. Increasing aggregate packing density and squeeze against one another result in a reduction in internal contact resistance as the fibre loading increases in density. As a result, the net resistance decreases as the level of fibre loading increases. There is too much variation in the fibre orientation for current to flow through it effectively. A large number of fibers in a small volume fraction results in the population of fibers being optimal for uniform dispersion and current flow.

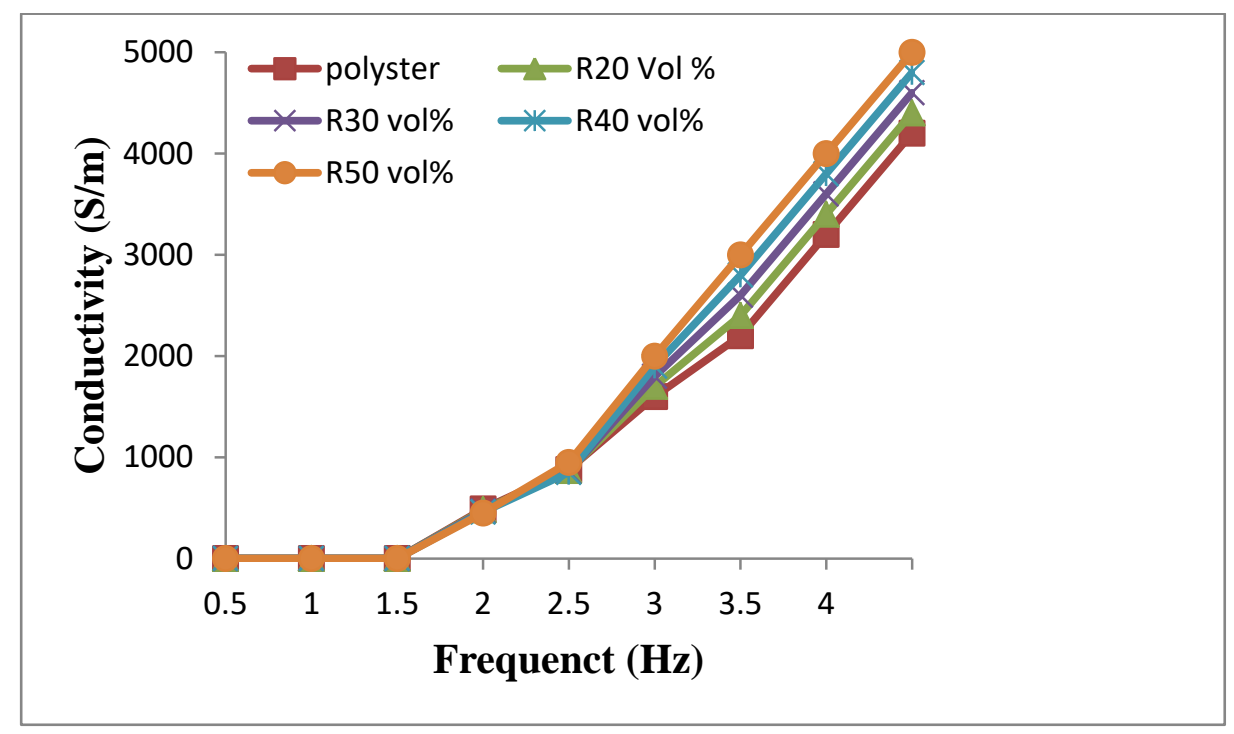

Figure 6.Electrical conductivity of the banana leaf composites with various fiber loading and different frequencies at $30^{\circ} \mathrm{C}$ 


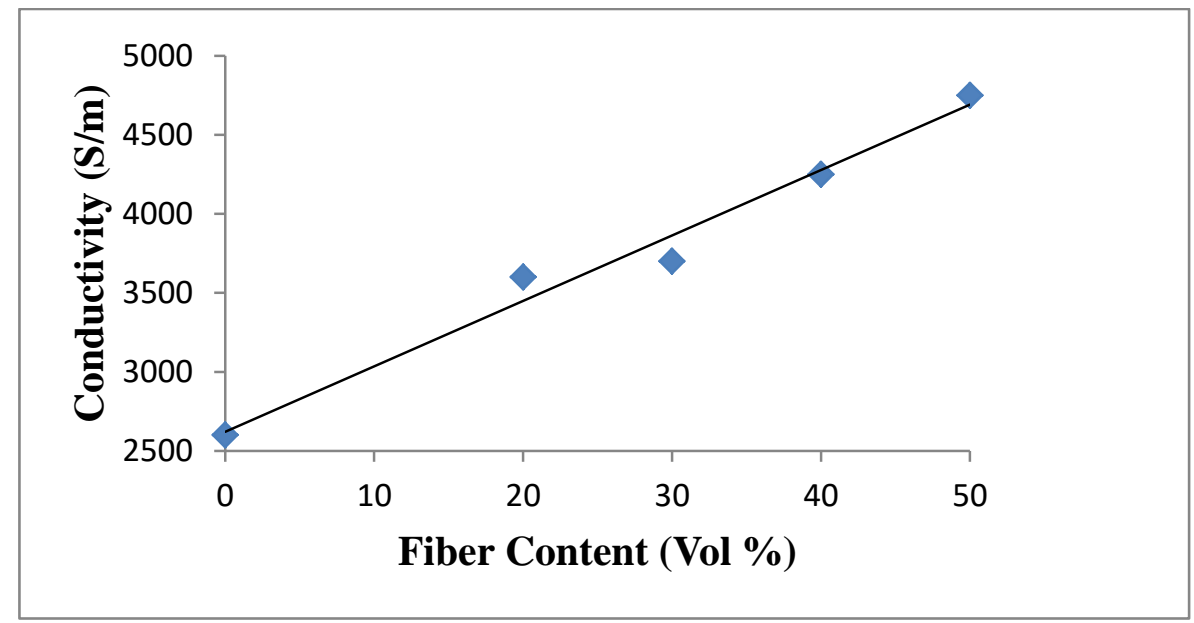

Figure 7.Electrical conductivity of banana leaf composites with fiber loading at lower frequency $(300 \mathrm{kHz})$ at $30^{\circ} \mathrm{C}$.

\subsection{EFFECT OF TEMPERATURE}

The effects of temperature on the dielectric constant of natural fibre reinforced composites are expected to manifest themselves in two ways. The first is the effect of temperature on the concentration of sobbed polar species, which are primarily composed of water. An additional characteristic of orientation polarizability is that it is affected by temperature (from permanent dipoles). As previously stated, the polarizability of a material is determined by its dielectric constant, which is composed of a variety of components, including electronic, atomic, orientation, and space charge. Only the orientation polarization (i.e., the contribution of the material's permanent dipoles) is affected by changes in the temperature. Figure 8 depicts the fluctuation in dielectric constant for banana leaf-polymer composites with varying fibre loading at a lower frequency than the previous Figure $(5 \mathrm{~Hz})$. As the temperature rises, the $\varepsilon_{0}$ values tend to increase in size in general. In RTM-produced composites, the $\varepsilon_{0}$ value increases at first and then decreases over time. The amount of variation is determined by the amount of fibre present. 


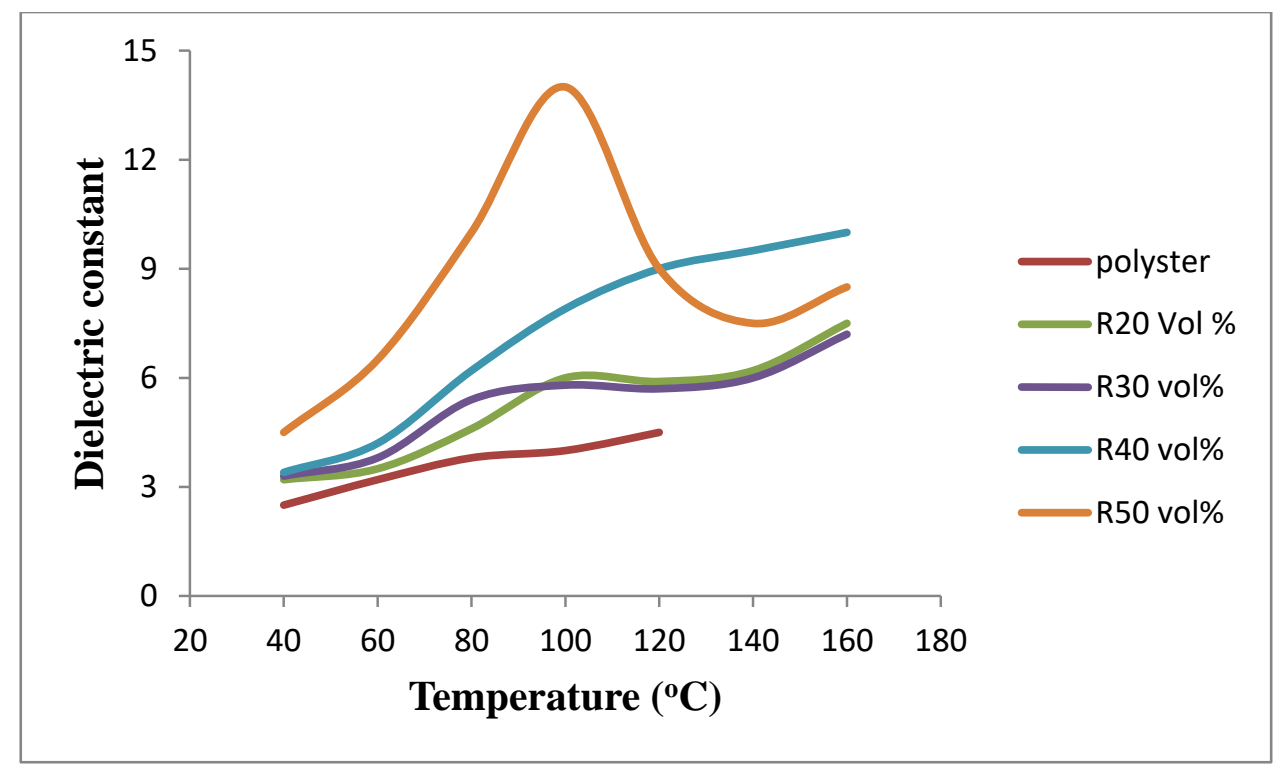

\section{Figure 8.Effect of fiber loading of banana leaf-Polymer composites with different temperatures}

Because the orientation of the fibers varies, this is most likely due to the fact that the overall physical and chemical texture of the composites varies as well. In addition, an increase in the mobility of water dipoles results in an increase in the $\varepsilon_{0}$ values of the polarization. Our previous research on the thermal stability of these composites demonstrated that increasing the fibre content of the composite results in an increase in the moisture content of the composite, as shown in the Figure [1].

\subsection{DIELECTRIC CONSTANT MODELING}

A linear relationship is shown in Figure. 9, shows that the reciprocal dielectric constant of composites $\left(1 / \dot{\varepsilon}_{\mathrm{c}}\right)$ plots against the volume percent of fibre is linear. This is the series combination of the dielectrics that make up the composites' constituents.

In the case of $\mathrm{V}_{\mathrm{f}}=0$, we obtain $1 / \varepsilon_{\mathrm{c}}=1 / \varepsilon_{\mathrm{m}}$ where $\dot{\varepsilon}_{\mathrm{m}}$ is the dielectric constant of the Polymer matrix at a frequency of $5 \mathrm{~Hz}$ and $\mathrm{c}$ is the length of the plot. There was only a slight difference between the empirically determined value (3.1) and the graphically determined value (3.1), which was discovered (3.4). On the graph in Figure 10, you can see how the composites' specific polarization varies in relation to their fibre content. 


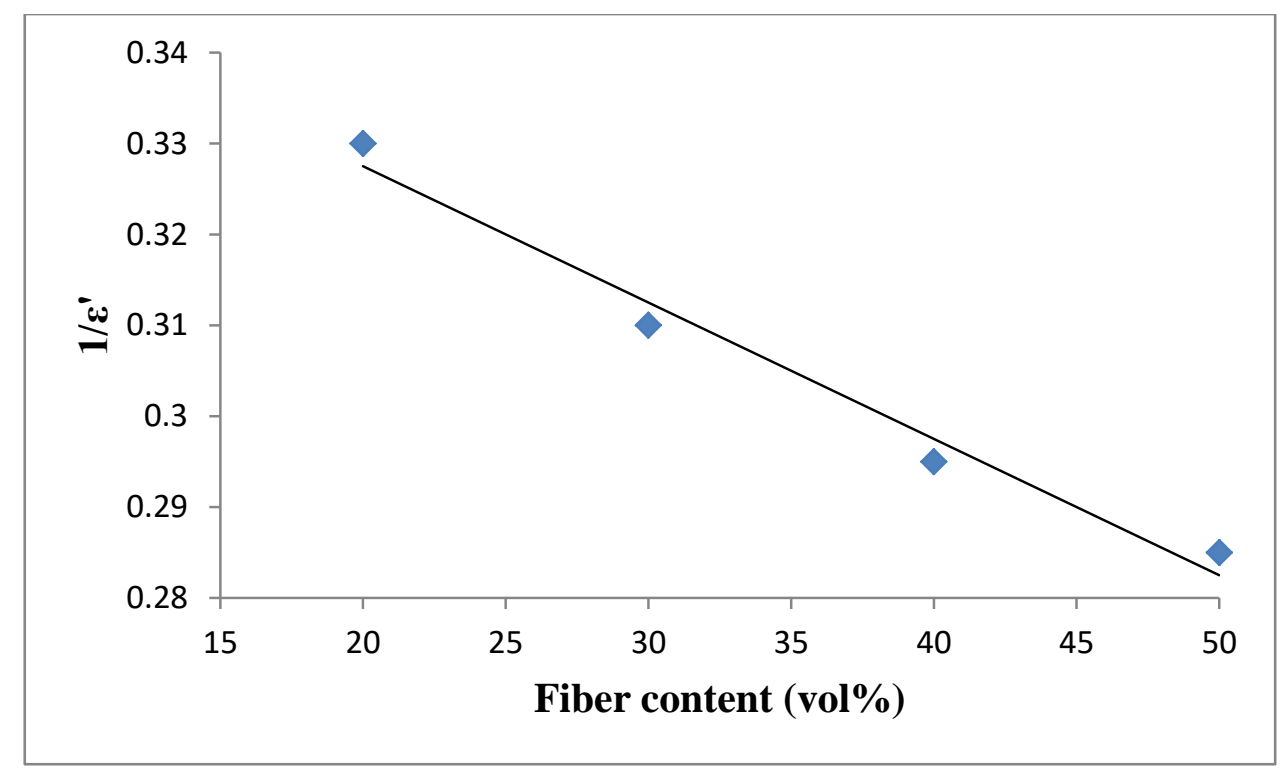

Figure 9.Reciprocal of dielectric constant of volume fraction of fiber with different frequencies of $5 \mathrm{~Hz}$ at $30^{\circ} \mathrm{C}$.

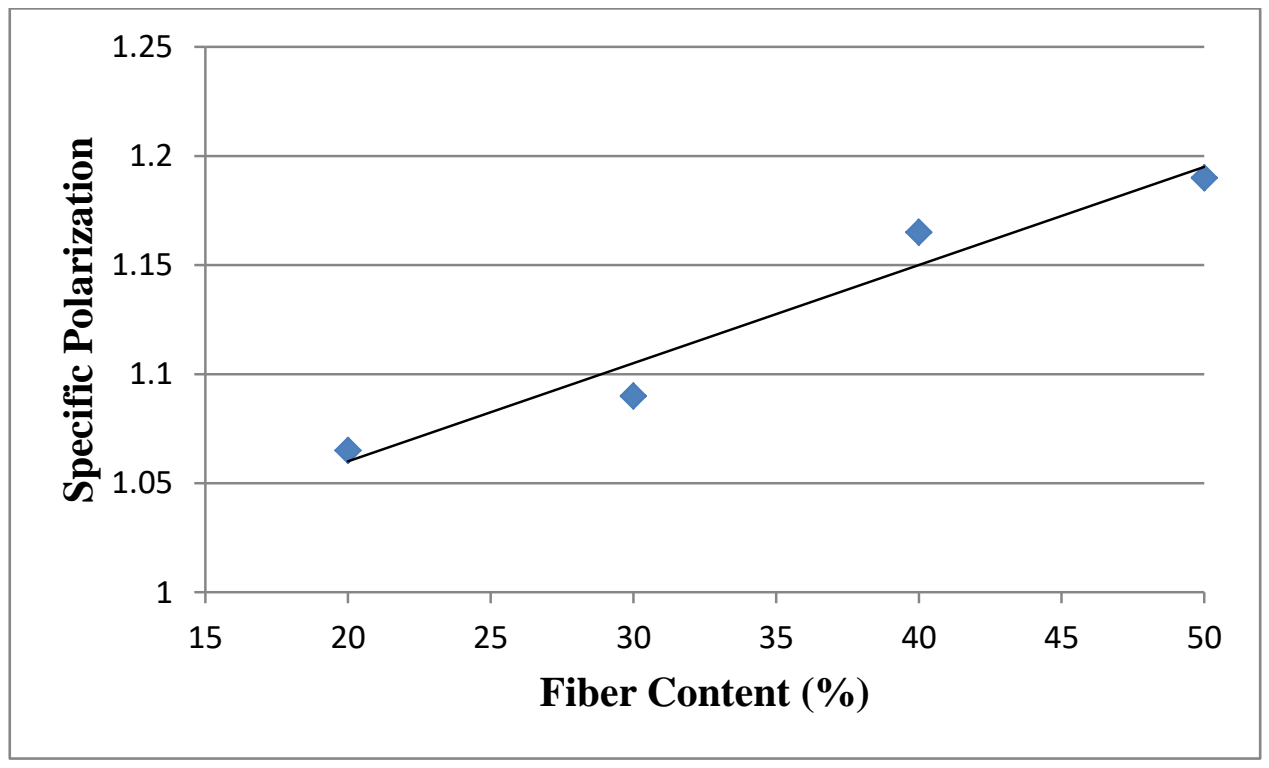

Figure 10.Specific polarization and volume fraction of banana leaf fiber loading $(5 \mathrm{~Hz})$ at $30^{\circ} \mathrm{C}$.

When specific polarization is plotted against fibre content, a linear pattern is obtained, which is consistent with the Clausius Mossotti Equation's prediction [1]. The intercepts of these charts at $\mathrm{V}_{\mathrm{f}}=0$ can be used to calculate the dielectric constant of the polymer using theoretical calculations. When the graph was extrapolated to the y axis, the intercept was 3.98, which was the same as the comparable experimental value of 3.98 in the original experiment. 


\section{CONCLUSION}

RTM's investigation into the electrical properties of banana leaf reinforced polymer composites revealed that increasing the amount of fibre in the polymer matrix increases the dielectric constant, loss factor, dissipation factor, and conductivity of the composites, among other things. It is more noticeable in composites that contain $50 \%$ or more natural fibers, due to the increase in orientation and interfacial polarizations caused by the presence of cellulose polar groups in natural fibers. Natural fibers have a higher orientation and interfacial polarization than synthetic fibers. Additionally, the presence of these polar groups allows current to flow within the polymer matrix, resulting in a decrease in the volume resistivity of the polymer material. Also discovered is that increasing the temperature of composites results in an increase in the dielectric constant values of the materials. It has been discovered that the measured values of the dielectric constant are within a small range of the values calculated using various theories of heterogeneous dielectric materials.

\section{REFERENCES}

[1] Chen, Xin, Hao, Jian, Liao, Ruijin, Li, Jian, Yang, Lijun and Feng, Dawei, “AC Surface Flashover and Gas Generation Difference of the Cellulose Insulation Pressboard Immersed in Novel 3-Element Mixed Oil and Mineral Oil", IEEE Access., Vol. 7, no.2, (2019), pp. 147048-147059.

[2] P.A. Sreekumar, Jean Marc Saiter, Kuruvilla Joseph, G. Unnikrishnan and Sabu Thomas, "Electrical properties of short sisal fiber reinforced Polymer composites fabricated by resin transfer molding", Composites Part A: Applied Science and Manufacturing., Vol.43, no.3, (2012), pp. $490-511$.

[3] Navin Chand, Deepak Jain and Archana Nigrawal, "Investigations on gradient a.c. conductivity characteristics of bamboo", Indian Academy of Sciences, Vol. 29 no.2, (2006), pp. 193-196.

[4] Peças, Paulo., Carvalho, Hugo, Salman, Hafiz and Leite, Marco, "Natural Fibre Composites and Their Applications: A Review", Journal of Composites Science, Vol. 2, no. 4, (2018), pp. 66-77.

[5] Li, Mi, Pu, Yunqiao, Thomas, M. Valerie M, Yoo, Chang Geun, Ozcan, Soydan, Deng., Yulin, Nelson, Kim and Ragauskas, J. Arthur. " Recent Advancements of Plant-Based 
Natural Fiberâ€ Reinforced Composites and Their Applications”, Composites Part B: Engineering, Vol. 7, no.4, (2020), pp.108254- 108268.

[6] M. Idicula, N.R. Neelakantan, Z. Oommen, K. Joseph, and S. Thomas, "A study of the mechanical properties of randomly oriented short banana and banana leaf hybrid fiber reinforced Polymer composites”, J Appl Polym Sci Vol 96, no. 5, (2005), pp. 1699709.

[7] M.S. Sreekala, G. Jayamol, M.G. Kumaran and S. Thomas, "The mechanical performance of hybrid phenol-formaldehyde-based composites reinforced with glass and oil palm fibers", Compos Sci Technol. Vol. 62, no.6, (2018), pp. 339-353.

[8] K.S. Ahmed and S. Vijayarangan, "Tensile, flexural and inter laminar shear properties of woven jute and jute-glass fabric reinforced Polymer composites”, J Mater Process Technol., Vol. 5 no. 2 (2015), pp. 330-335.

[9] N. Liang, R. Liao, M. Xiang, Y. Mo and Y. Yuan, "Influence of amine compounds on the thermal stability of paper-oil insulation Polymers"., Vol. 10, no. 8, (2018), pp. 891 917.

[10] L.A. Pothan, Y.W. Mai, S. Thomas and R.K.Y. Li, "Tensile and flexural behavior of banana leaf fabric-Polymer textile composites prepared by resin transfer molding technique”, J Reinf Plast Compos., Vol. 27, no. 5, pp. 1847-66.

[11] G. Francucci, E. Rodriguez, and A. Vázquez, "Study of saturated and unsaturated permeability in natural fiber fabrics”, Compos Part A: Appl Sci and Manuf., Vol. 41 no. 4, (2010), pp. 16-21.

[12] A.N. Towo and M.P. Ansell, "Fatigue evaluation and dynamic mechanical thermal analysis of banana leaf fiber-thermosetting resin composites", Compos Sci Technol., Vol. 68, no. 3, (2008), pp. 925-32.

[13] Rajak, Dipen Kumar., Pagar, D. Durgesh, Kumar, Ravinder., Pruncu, Catalin.” Recent progress of reinforcement materials: a comprehensive overview of composite materials", Journal of Materials Research and Technology., Vol. 16, no. 3, (2019), pp. $2086-2087$.

[14] F.H. Gojnya, M.H.G. Wichmann, B. Fiedler, W. Bauhofer, and K. Schulte, "Influence of nano-modification on the mechanical and electrical properties of conventional fiberreinforced composites”, Compos Part A: Appl Sci Manuf., Vol. 36, no. 2, (2008), pp.1525-1535.

[15] Wu, SL, Tung, "IC. Dielectric studies of mineral-filled epoxy", Polym Compos., Vol. 6, no. 3, (2010), PP, 233-299. 
[16] M.A.G. Martins, "Vegetable oils an alternative to mineral oil for power transformersexperimental study of paper aging in vegetable oil versus mineral oil”, IEEE Elect. Insul. Mag., Vol. 26, no. 6 (2010, pp. 7 -13.

[17] J. Liu, X. Fan, H. Zheng, Y. Zhang, C.Zhang, B. Lai., J. Wang, G. Ren, and E. Zhang, "Aging condition assessment of transformer oil-immersed cellulosic insulation based upon the average activation energy method", Cellulose., Vol. 26, no.6, (2019), pp 3891 -3908 .

[18] N. Hayakawa, T. Kobayashi, M. Hazeyama, T. Takahashi, K. Yasuda, and H. Okubo, “ Partial discharge inception characteristics of LN2-PP laminated paper composite insulation system for high temperature superconducting cables", IEEE Trans. Dielectr. Electr. Insul. Vol. 12, no. 4, (2019), pp. 166-174.

[19] S. Joseph, and S. Thomas, "Electrical properties of banana fiber-reinforced phenol formaldehyde composites”, J Appl Polym Sci., Vol. 63, no. 2, (2009), pp. 256 - 63.

[20] N. Chand,. and D. Jain, "Effect of banana leaf fiber orientation on electrical properties of banana leaf fiber reinforced epoxy composites", Compos Part A: Appl Sci Manuf.. Vol. 36, no. 3, (2005), pp. 594-602.

[21] E. Rodríguez, R. Petrucci, D., Puglia, J. Kenny, and A. Vázquez,, "Characterization of different composites based on natural and glass fibers obtained by vacuum infusion"., J Compos Mater., Vol. 39, no. 4, (2005), pp. 265-82.

[22] E. Rodríguez, F. Giacomelli, and A. Vázquez, 2004, "Permeability-porosity relationship in RTM for different fiberglass and natural reinforcements", Journal J Compos Mater., Vol. 38, no. 2, (2006), pp. 259-68.

[23] V.G. Geethamma, G. Kalaprasad, G. Groeninckx, and S. Thomas, "Dynamic mechanical behavior of short coir fiber reinforced natural rubber composites", Compos Part A: Appl Sci Manuf., Vol. 36, no. 3, (2005), pp.1499-506. 